Running title: Metal-Insulator Transition in Doubly Degenerate Hubbard's model

The Metal-Insulator Transition

\title{
in the Doubly Degenerate Hubbard Model
}

\author{
Hideo Hasegawa ${ }^{\dagger}$ \\ Department of Physics, Tokyo Gakugei University \\ Koganei, Tokyo 184, Japan
}

(Received April 28, 1997)

\begin{abstract}
A systematic study has been made on the metal-insulator (MI) transition of the doubly degenerate Hubbard model (DHM) in the paramagnetic ground state, by using the slaveboson mean-field theory which is equivalent to the Gutzwiller approximation (GA). For the case of infinite electron-electron interactions, we obtain the analytic solution, which becomes exact in the limit of infinite spatial dimension. On the contrary, the finiteinteraction case is investigated by numerical methods using the simple-cubic model with the nearest-neighbor hopping. The mass-enhancement factor, $Z$, is shown to increase divergently as one approaches the integer fillings $(N=1,2,3)$, at which the MI transition takes place, $N$ being the total number of electrons. The calculated $N$ dependence of $Z$ is compared with the observed specific-heat coefficient, $\gamma$, of $\mathrm{Sr}_{1-x} \mathrm{La}_{x} \mathrm{TiO}_{3}$ which is reported to significantly increase as $x$ approaches unity.
\end{abstract}

Keywords: slave boson, Gutzwiller approximation, metal-insulator transition

$\dagger$ e-mail address: hasegawa@u-gakugei.ac.jp 


\section{$\S 1$. Introduction}

A study on the metal-insulator (MI) transition has been one of the most fascinating subjects in solid state physics. ${ }^{1)}$ In recent years much attention has been paid to transition-metal compounds of $\mathrm{A}_{1-x} \mathrm{~B}_{x} \mathrm{MO}_{3}$ where $\mathrm{A}=\mathrm{Sr}, \mathrm{Ca}, \mathrm{B}=\mathrm{La}, \mathrm{Y}$, and $\mathrm{M}=\mathrm{Ti}$, V, Cr. ${ }^{2,3)}$ These systems exhibit various phases such as a paramagnetic metal (PM), paramagnetic insulator (PI) and antiferromagnetic insulator (AFI) when the temperature $(T)$, pressure $(P)$, magnetic field $(H)$ or chemical substitution $(x)$ is changed. Theoretically the MI transition has been so far investigated by using the single-band Hubbard model $(\mathrm{SHM}),{ }^{4-6)}$ with which observed $T-P$ and $T-x$ phase diagrams have been qualitatively understood. ${ }^{7-12)}$ We should, however, remind the fact that constituent transition metals of these systems inevitably have the orbital degeneracy. Although the important role played by the orbital degeneracy has been recognized, the additional degree of the orbital degeneracy has prevented us from a systematic, theoretical analysis using the degenerate-band Hubbard model.

In the last few years, several theoretical studies have been made of the MI transition in the Hubbard model with orbital degeneracy, by employing various methods such as the Gutzwiller approximation (GA), ${ }^{13-16)}$ Monte-Carlo method ${ }^{17-19)}$ and slave-boson theory. ${ }^{20-22)}$ By using the $\mathrm{GA}, \mathrm{Lu}^{13)}$ obtained the critical interaction for the MI transition, $U_{c}$, given by $U_{c} \sim(D+1)$ at the half filling, while Gunnarsson et al. ${ }^{17)}$ showed $U_{c} \sim \sqrt{ } D$, using a diffusion Monte Carlo method, D denoting the orbital degeneracy. Bünemann and Weber ${ }^{14)}$ discussed the first-order MI transition in the half-filled doubly degenerate Hubbard model (DHM) when the exchange interaction is included within the GA. The MI transition of the infinite-dimensional DHM in the paramagnetic state is investigated 
by Kotliar and Kajueter, ${ }^{18)}$ and by Rozenberg ${ }^{19)}$ using the dynamical mean-field theory.

In a previous paper (referred to as I), ${ }^{20)}$ the present author proposed the slaveboson functional-integral theory for the degenerate-band Hubbard model, by adopting the method originally employed for the Anderson lattice model. ${ }^{23}$ It is a generalization of the slave-boson method of the $\mathrm{SHM}^{24)}$ to the degenerate-band model, and its mean-field approximation is equivalent to the GA, ${ }^{13-15,25)}$ which becomes exact for the Gutzwiller wavefunctions in the limit of the infinite dimension. ${ }^{16,26)}$ An alternative slaveboson method was independently proposed by Fresard and Kotliar. ${ }^{21)}$ In our second paper (referred to as II ${ }^{22)}$ the antiferromagnetic state of the half-filled DHM was studied by using our slave-boson theory. ${ }^{20)}$

Although these studies ${ }^{13-22)}$ are useful in understanding the existing experimental data, it is more desirable to make a systematic study of the MI transition of the degenerateband Hubbard model. We have made such a study by using the slave-boson mean-field theory developed in I, which is the purpose of the present paper. In the next $\S 2$, we briefly discuss the mean-field approximation to our slave-boson theory ${ }^{20)}$ applied to the DHM. One of the advantages of our slave-boson mean-field theory over sophisticated methods such as the dynamical mean-field method, ${ }^{18,19)}$ is that we can obtain analytic solutions in some limiting cases. Indeed, we present, in $§ 3$, the analytic solution when the electronelectron interactions are infinite. For finite interactions, we perform, in $\S 4$, numerical calculations using the simple-cubic model. The final section $(\S 5)$ is devoted to conclusion and discussion on the experimental data of $\mathrm{Sr}_{1-x} \mathrm{La}_{x} \mathrm{TiO}_{3} \cdot{ }^{3)}$

\section{$\S 2$. Formulation}


We adopt the DHM whose Hamiltonian is given by

$$
H=\sum_{\sigma} \sum_{i j} \sum_{m m^{\prime}} t_{i j}^{m m^{\prime}} c_{i m \sigma}^{\dagger} c_{j m^{\prime} \sigma}+\frac{1}{2} \sum_{i} \sum_{(m, \sigma) \neq\left(m^{\prime}, \sigma^{\prime}\right)} U_{m m^{\prime}}^{\sigma \sigma^{\prime}} c_{i m \sigma}^{\dagger} c_{i m \sigma} c_{i m^{\prime} \sigma^{\prime}}^{\dagger} c_{i m^{\prime} \sigma^{\prime}}
$$

where $c_{i m \sigma}$ is an annihilation operator of an electron with an orbital index $m$ and spin $\sigma(=\uparrow, \downarrow)$ on the lattice site $i$. The electron hopping is assumed to be allowed only between the same sub-band: $t_{i j}^{m m^{\prime}}=t_{i j} \delta_{m m^{\prime}}$ for a simplicity. The on-site interaction, $U_{m m^{\prime}}^{\sigma \sigma^{\prime}}$, is given by

$$
\begin{aligned}
U_{m m^{\prime}}^{\sigma \sigma^{\prime}} & =U_{0}=U \quad \text { for } m=m^{\prime}, \sigma \neq \sigma^{\prime}, \\
& =U_{1}=U-2 J \text { for } m \neq m^{\prime}, \sigma \neq \sigma^{\prime}, \\
& =U_{2}=U-3 J \text { for } m \neq m^{\prime}, \sigma=\sigma^{\prime},
\end{aligned}
$$

where $U$ and $J$ are Coulomb and exchange interactions, respectively.

For the paramagnetic state of the DHM, we introduce the boson operators of $e_{i}, p_{i}$, $d_{i}, t_{i}$ and $f_{i}$ which denote the empty, singly-, doubly-, triply- and fully-occupied states, respectively, at a given $i$ site. $^{20,22)}$ As for the doubly occupied states, we take into account three kinds of configurations; $d_{i 0}$ for a pair of electrons on the same orbital with opposite spin, $d_{i 1}$ on the different orbital with opposite spin, and $d_{i 2}$ on the different orbital with same spin. These boson operators are under constraints of their completeness and the correspondence with fermion operators. ${ }^{20,22)}$

When we apply our slave-boson mean-field theory developed in I to the DHM under consideration, we get the ground-state energy given by

$$
E=E_{1}+E_{2}
$$


where

$$
\begin{aligned}
& E_{1}=2 q \varepsilon_{0}, \\
& E_{2}=2\left(U_{0} d_{0}+U_{1} d_{1}+U_{2} d_{2}\right)+2\left(U_{0}+U_{1}+U_{2}\right)(2 t+f) .
\end{aligned}
$$

Here the occupancies of $d_{0}, d_{1}, d_{2}, t$ and $f$ are the expectation values of respective boson operators and they are determined by the following self-consistent equations: ${ }^{20,22)}$

$$
\begin{gathered}
U_{0}+\varepsilon_{0}\left(\partial q / \partial d_{0}\right)=0, \\
U_{1}+\varepsilon_{0}\left(\partial q / \partial d_{1}\right)=0, \\
U_{2}+\varepsilon_{0}\left(\partial q / \partial d_{2}\right)=0, \\
2\left(U_{0}+U_{1}+U_{2}\right)+\varepsilon_{0}(\partial q / \partial t)=0, \\
\left(U_{0}+U_{1}+U_{2}\right)+\varepsilon_{0}(\partial q / \partial f)=0 .
\end{gathered}
$$

where

$$
\begin{gathered}
q=\frac{\left.16\left[\left(\sqrt{d_{0}}+\sqrt{d_{1}}+\sqrt{d_{2}}\right)(\sqrt{p}+\sqrt{t})+\sqrt{\overline{e p}}+\sqrt{t f}\right)\right]^{2}}{N(4-N)}, \\
\varepsilon_{0}=\int^{\mu} \mathrm{d} \varepsilon \sum_{\sigma} \varepsilon \rho_{0}(\varepsilon) \\
N=2 \int^{\mu} \mathrm{d} \varepsilon \sum_{\sigma} \rho_{0}(\varepsilon)
\end{gathered}
$$

with

$$
\begin{gathered}
e=1-N+2\left(d_{0}+d_{1}+d_{2}\right)+8 t+3 f, \\
p=N / 4-\left(d_{0}+d_{1}+d_{2}\right)-3 t-f .
\end{gathered}
$$

In eqs. (7)-(16), $\mu$ is the Fermi level, $\rho_{0}(\varepsilon)$ denotes the non-interacting density of states per sub-band per spin, and $N$ is the total number of electrons which is 1 and 2 for the 
quarter- and half-filled bands, respectively. The band-narrowing factor, $q$, is expressed in terms of various occupancies as given by eq. (12). The self-consistent equations given by eqs. (7)-(16) are equivalent to those obtained by employing the GA to Gutzwiller's wavefunctions. ${ }^{13-15)}$

\section{$\S 3$. Analytic Solutions for Infinite Interactions}

When the interactions are infinite, we can obtain analytic solutions for the selfconsistent equations given by eqs. (7)-(16). Solutions for the less-than-half filling $(N \leq 2)$ are classified into the three cases depending on the value of $j$ defined by

$$
j=\lim _{U \rightarrow \infty}(J / U)
$$

Solutions for the more-than-half filling $(N>2)$ can be obtained from the result for $N \leq 2$ with the electron-hole symmetry.

\subsection{Case of $j=0$}

When $j=0$, three, double occupancies become equivalent: $d_{0}=d_{1}=d_{2}=d$. Taking the $U \rightarrow \infty$ limit of eq. (7), (8) or (9) with

$$
t=f=0
$$

we get

$$
\begin{aligned}
d_{0}=d_{1}=d_{2} & =0 & & (\text { for } N \leq 1), \\
& =(1 / 6)(N-1) & & (\text { for } 1<N<2) .
\end{aligned}
$$

From eqs. (18)-(20), $e$ and $p$ in eqs. (15) and (16) are given by

$$
e=1-N \quad(\text { for } N \leq 1)
$$




$$
\begin{array}{cr}
=0 & (\text { for } 1<N \leq 2) . \\
p=N / 4 & (\text { for } N \leq 1), \\
=(1 / 4)(2-N) & (\text { for } 1<N \leq 2) .
\end{array}
$$

Substituting eqs. (18)-(24) to eq. (12), we obtain the band-narrowing factor:

$$
\begin{aligned}
q & =4(1-N) /(4-N) & & (\text { for } N \leq 1), \\
& =6(N-1)(2-N) / N(4-N) & & (\text { for } 1<N \leq 2) .
\end{aligned}
$$

Near $N=1$ and $2, q$ is given by

$$
\begin{aligned}
q & =(4 / 3) \delta & & (\text { for } N \lesssim 1), \\
& =2 \delta & & (\text { for } N \gtrsim 1), \\
& =(3 / 2) \delta & & (\text { for } N \lesssim 2),
\end{aligned}
$$

where the electron or hole doping concentration, $\delta$, is given by $\delta=|1-N|$ in eqs. (27) and (28) and $\delta=|2-N|$ in eq. (29).

3.2 Case of $j>0$

Similarly we get

$$
\begin{array}{ll} 
& d_{0}=d_{1}=t=f=0, \\
d_{2}=0 & (\text { for } N \leq 1), \\
=(1 / 2)(N-1) & (\text { for } 1<N \leq 2) .
\end{array}
$$

The $N$-dependence of $e$ and $p$ is given by eqs. (21)-(24). The band-narrowing factor becomes

$$
q=4(1-N) /(4-N) \quad(\text { for } N \leq 1),
$$




$$
=2(N-1)(2-N) / N(4-N) \quad(\text { for } 1<N \leq 2) .
$$

It is easy to see that $q$ close to $N=1$ and 2 is given by

$$
\begin{aligned}
q & =(4 / 3) \delta & & (\text { for } N \lesssim 1), \\
& =(2 / 3) \delta & & (\text { for } N \gtrsim 1), \\
& =(1 / 2) \delta & & (\text { for } N \lesssim 2),
\end{aligned}
$$

where $\delta=|1-N|$ in eqs. (35) and (36) and $\delta=|2-N|$ in eq. (37).

3.3 Case of $j<0$

We get $^{27)}$

$$
\begin{array}{cc} 
& d_{1}=d_{2}=t=f=0, \\
d_{0}=0 & (\text { for } N \leq 1), \\
=(1 / 2)(N-1) & (\text { for } 1<N \leq 2) .
\end{array}
$$

The $N$-dependences of $e, p$ and $q$ are same as in the $j>0$ case.

It is worth to remark that in the SHM, the solution for $U \rightarrow \infty$ with $N \leq 1$ is given by

$$
\begin{gathered}
e=1-N, \\
p=N / 2,
\end{gathered}
$$

with $d=0$, leading to

$$
\begin{aligned}
q & =2(1-N) /(2-N) & \\
& \sim 2 \delta & (\text { for } N \lesssim 1),
\end{aligned}
$$


where $\delta=|1-N|$. We should note that these solutions of the GA for infinite interactions discussed above become exact in the limit of the infinite spatial dimension. ${ }^{16,26)}$ Some figures depicting the $N$ dependence of the occupancies and the band-narrowing factor for the DHM will be shown shortly (dashed curves in Figs. 3 - 5).

\section{$\S 4$. Numerical Calculations for Finite Interactions}

When the interactions are finite, we cannot obtain the analytic solution for the selfconsistent equations given by eqs. (7)-(16), which have to be solved by numerical methods for given parameters of $N, U$ and $J$ and non-interacting density of states, $\rho_{0}(\varepsilon)$. We adopt the simple-cubic model band, expressed by an approximate, analytic form: ${ }^{28)}$

$$
\begin{aligned}
\rho_{0}(\varepsilon) & =A\left[9-\omega^{2}\right]^{1 / 2}-C\left[1-\omega^{2}\right]^{1 / 2} \quad \text { for }|\omega| \leq 1, \\
& =A\left[9-\omega^{2}\right]^{1 / 2}-B\left[1-(|\omega|-2)^{2}\right]^{1 / 2}, \quad \text { for } 1<|\omega| \leq 3, \\
& =0 \quad \text { for }|\omega|>3,
\end{aligned}
$$

with $\omega=\varepsilon / 2 t, A / 2 t=0.101081, B / 2 t=0.128067$ and $C / 2 t=0.02, t$ being the nearestneighbor hopping. The energy and the interactions are hereafter measured in units of a half of the total band width $(W / 2=6 t=1)$.

\subsection{Results as a function of $U$}

Firstly we discuss the $U$ dependence of the band-narrowing factor, $q$, at the integer fillings of $N=1$ and $2{ }^{29)}$ Figure 1 (a) shows the $U$ dependence of $q$ for various values of $J / U$ of the quarter-filled case $(N=1)$. When the $U$ is increased, the $q$ factor decreases and vanishes above $U_{c}$ which stands for the critical interaction for the MI transition. We get $q \sim\left(U_{c}-U\right)$ at $U \leq U_{c}$, which is the same as in the SHM. ${ }^{30)}$ In all the cases shown 
in Fig. 1(a), the MI transition is of the second order. The calculated $U_{C}$ is plotted by the solid curve in Fig. 2 as a function of $J / U$, which shows an increase in $U_{c}$ as increasing $J / U$. On the contrary, the behavior of the MI transition in the half-filled band $(N=2)$ is quite different from that in the $N=1$ case, as is shown in Fig. 1(b). The MI transition becomes the first-order one for finite $J / U,{ }^{14,20)}$ and $U_{c}$ decreases as increasing the absolute value of $J / U$. Then the $U_{c}$ curve has a cusp at $J / U=0$, as shown by the dashed curve in Fig. 2.

\subsection{Results as a function of $N$}

Figures 3(a) shows the $N$ dependence of the occupancies for various $U$ values with $J / U=0$. At $N=0$ only the empty occupancy $e$ is finite $(e=1)$. As increasing $N$ from zero, $e$ monotonously decreases while $p$ has a peak at $N=1$, where the double occupancies, $d_{0}=d_{1}=d_{2}=d$, begin to increase. Since $t$ and $f$ are one or two-order magnitude smaller than the other occupancies, they are not shown in the Figure. Dashed curves express analytic solutions of $e, p$ and $d$ for $U=\infty$ given by eqs. (18)-(24). We note that as the $U$ value is increased, the $N$ dependence of the occupancies approaches that for $U=\infty$ and that the occupancies for $U=4$ are almost the same as those for $U=\infty$

Similarly, the $N$ dependence of the occupancies for various $U$ values with $J / U=0.1$ is shown in Fig. 3(b). General behavior for $J / U=0.1$ is very similar to that for $J / U=0$, except that only $d_{2}$ has an appreciable magnitude among the three, double occupancies because $U_{0}>U_{1}>U_{2}$ for $J>0$.

Figures 4(a) and 4(b) show $q$ as a function of $N$ for various $U$ with $J / U=0$ and 
$J / U=0.1$, respectively. The band-narrowing factor is unity at $N=0$, and it has dips or zeros at $N=1$ and 2. Our result of the $N$ dependence of $q$ for $J / U=0$ is similar to those reported in refs. 19 and 21. The mass-enhancement factor, $Z$, which is given as the inverse of $q$, for $J / U=0$ and $J / U=0.1$ is shown in Figs. 5(a) and 5(b), respectively, as a function of $N$. For intermediate interactions $(U \sim W), Z$ has peaks at the integer band fillings of $N=1$ and 2, where $Z$ shows the divergence for the strong interaction $(U \gg W)$. As increasing the $U$ value, $Z$ increases as expected. Dashed curves in Figs. 5(a) and 5(b) express the results for $U=\infty$ given by eqs. (25)-(26) and (33)-(34). We note that $Z$ at $N \leq 1$ is not symmetric with that at $N>1$ and it is not the same as that at $N \leq 2$, in particular for $J / U=0.1$. This is more clearly seen in the case of $U=\infty$ as shown by dashed curves in Figs. 5(a) and 5(b) and as given by eqs. (27)-(29) and (35)-(37), which show the $\delta^{-1}$ divergence as approaching the integer fillings.

\section{$\S 5$. Conclusion and Discussion}

By using the slave-boson mean-field theory ${ }^{20)}$ which is equivalent to the GA, ${ }^{13-16,25)}$ we have systematically studied the DHM, which shows the MI transition to take place at the integer fillings of $N=1,2$ and 3 in the paramagnetic state. When the magnetically ordered state is taken into account, this MI transition is modified, particularly in the half-filling case, where the antiferromagnetic state can be realized for a relatively small interaction. Indeed, for the simple-cubic model with the nearest-neighbor hopping, the antiferromagnetic insulator (AFI) is stabilized for an infinitesimally small interaction, and then the MI transition does not take place except at the vanishing interaction point in the DHM, ${ }^{22)}$ just as in the SHM. ${ }^{24,31)}$ The schematic, phase diagram of the MI transition 
of the DHM is shown in Fig. 6. When approaching the integer filling from below or above with the moderate interaction $(U \sim W)$, the mass-enhancement factor, $Z$, is considerably increased at the PM-PI transition. On the contrary, when the PM-AFI (PM-FI) transition occurs with the strong interaction and/or the favorable band filling (as the half filling), the mass-enhancement is not realized in the AFI (FI) because of the presence of the energy gap in the density of states, ${ }^{32)}$ although the enhancement of $Z$ is expected as approaching this transition from the metallic side. Our calculations applying the slave-boson meanfield theory to the DHM, ${ }^{20,22)}$ have well accounted for the essential features of the MI transition of a system with orbital degeneracy:

It has been reported that the linear coefficient of the low-temperature specific heat, $\gamma$, and the susceptibility of $\mathrm{Sr}_{1-x} \mathrm{La}_{x} \mathrm{TiO}_{3}$ significantly increase as $x$ approaches unity. ${ }^{3)}$ Filled circles in Figs. 5(a) and 5(b) express the observed mass-enhancement ratio of $\gamma / \gamma_{0}(=Z)$. We note that the calculated $N$ dependence of $Z$ well reproduces the observed $x$ dependence of $\gamma / \gamma_{0}$. It was previously tried to explain the experimental data with the use of the SHM, ${ }^{12)}$ in which the mass-enhancement factor, $Z$, of its paramagnetic state divergently increases near the half filling (eqs. (43) and (44)). We should, however, remind the fact that this enhancement does not occur if the antiferromagnetic state is included, as was discussed above (Fig. 6). For a better understanding of the MI transition in $\mathrm{Sr}_{1-x} \mathrm{La}_{x} \mathrm{TiO}_{3}$, we have to adopt the triply degenerate Hubbard model in a strict sense, because $d$ electrons in this system belong to $t_{2 g}$ bands. ${ }^{3)}$ In order to discuss the high-energy phenomena of transition-metal compounds ${ }^{2,3)}$ such as photoemission and the optical conductivity, it is necessary to take into account "incoherent-quasiparticle" states arising from boson fluctuations around the saddle-point solution: ${ }^{33)}$ our slave-boson mean-field 
theory includes only "coherent-quasiparticle" states, which are relevant to the low-energy phenomena like the mass enhancement. Going beyond the mean-field approximation is left as one of our future subjects in the slave-boson functional integral theory for the degenerate-band Hubbard model.

\section{Acknowledgment}

This work is partly supported by a Grant-in-Aid for Scientific Research from the Japanese Ministry of Education, Science and Culture. 


\section{References}

[1] N. F. Mott: Metal-Insulator Transitions, (Taylor \& Francis, London 1990) 2nd ed.

[2] A. Fujimori, I. Hase, H. Namatame, Y. Fujishima, Y. Tokura, H. Eisaki, S. Uchida, K. Takegawara, and F. M. F. de Groot: Phys. Rev. Lett. 69 (1992) 1796.

[3] Y. Tokura, Y. Taguchi, Y. Okada, Y. Fujishima, T. Arima, K. Kumagai, and Y. Iye: Phys. Rev. Lett. 70 (1993) 2126.

[4] M. C. Gutzwiller: Phys. Rev. Lett. 10 (1963) 159; Phys. Rev. 137 (1965) A1726.

[5] J. Kanamori: Prog. Theor. Phys. 30 (1963) 235.

[6] J. Hubbard: Proc. Roy. Soc. London A281 (1964) 401.

[7] T. Moriya and H. Hasegawa: J. Phys. Soc. Jpn. 48 (1980) 1490.

[8] H. Hasegawa: J. Phys. Soc. Jpn. 49 (1980) 178; Y. Kakehashi and H. Hasegawa: Phys. Rev. B 36 (1987) R4066.

[9] X. Y. Zhang, M. J. Rozenberg, and G. Kotliar: Phys. Rev. Lett. 70 (1993) 1666.

[10] Th. Pruschke, D. L. Cox and M. Jarrell: Phys. Rev. B 47 (1993) 3553.

[11] A. Georges and W. Krauth: Phys. Rev. B 48 (1993) 7167.

[12] M. J. Rozenberg, G. Kotliar and X. Y. Zhang: Phys. Rev. B 49 (1994) 10181.

[13] J. P. Lu: Phys. Rev. 49 (1994) 5687.

[14] J. Bünemann and W. Weber: Phys. Rev. B 55 (1997) R4011. 
[15] T. Okabe: J. Phys. Soc. Jpn. 65 (1996) 1056.

[16] J. Bünemann, F. Gebhard, and W. Weber. e-print (cond-mat/9704037).

[17] O. Gunnarsson, E. Koch, and R. Martin: Phys. Rev. B 54 (1966) R11026; preprint

[18] G. Kotliar and H. Kajueter: Phys. Rev. 54 (1996) R14221; e-perint (condmat/9609176).

[19] M. J. Rozenberg: Phys. Rev. B 55 (1997) 4855; e-print cond-mat/9612089).

[20] H. Hasegawa: J. Phys. Soc. Jpn. 66 (1997) 1391.

[21] R. Fresard and G. Kotliar: e-print (cond-mat/9712172).

[22] H. Hasegawa: Phys. Rev. B 56 (1997) 1196.

[23] V. Dorin and P. Schlottmann: Phys. Rev. 47 (1993) 5095.

[24] G. Kotliar and A. E. Ruckenstein: Phys. Rev. Lett. 57 (1986) 1362.

[25] K. A. Chao and M. C. Gutzwiller: Phys. Rev. B 4 (1971) 4034; K. A. Chao: J. Appl. Phys. 42 (1971) 1420.

[26] W. Metzner and D. Vollhardt: Phys. Rev. Lett. 59 (1987) 121.

[27] Although the exchange interaction $J$ is conventionally believed to be positive, we include the negative- $J$ case for a completeness of our discussion.

[28] T. Tonegawa: Prog. Theor. Phys. 51 (1974) 1293.

[29] Some calculated results of the $U$ dependence of the occupancies in the paramagnetic DHM have been reported in ref. 20 . 
[30] W. F. Brinkman and T. M. Rice: Phys. Rev. B 2 (1970) 4302.

[31] H. Hasegawa: J. Phys. Cond. Matter. 1 (1989) 9325; Phys. Rev. 41 (1990) 9168.

[32] M. Imada: J. Phys. Soc. Jpn. 62 (1993) 1105; In this reference the possibility of the two types of the MI transitions: (1) $Z \rightarrow \infty$ as $\delta \rightarrow 0$, and (2) $Z \rightarrow 0$ as $\delta \rightarrow 0$, is pointed out in the different context from ours, $\delta$ being the electron or hole concentration doped to the integer filling.

[33] R. Raimondi and C. Castellani: Phys. Rev. 48 (1993) R11453; R. Raimondi: Phys. Rev. 51 (1995) 10154. 


\section{Figure Captions}

Fig. 1 The $U$ dependence of the band-narrowing factor, $q$, for various $J / U$ with (a) $N=1$ and $(\mathrm{b}) N=2$.

Fig. 2 The critical interaction for the MI transition, $U_{c}$, in the paramagnetic state as a function of $J / U$ for $N=1$ and 2 , the solid (dashed) curve denoting the second (first) order transition.

Fig. 3 The $N$ dependence of the occupancies for $U=2$ (thin solid curve), 4 (bold solid curve) and $\infty$ (dashed curve) with (a) $J / U=0$ and (b) $J / U=0.1$.

Fig. 4 The $N$ dependence of the band narrowing factor, $q$, for various $U$ with (a) $J / U=0$ and (b) $J / U=0.1$, the dashed curve denoting the result for $U=\infty$.

Fig. 5 The $N$ dependence of the mass-enhancement factor, $Z(=1 / q)$, for various $U$ with (a) $J / U=0$ and (b) $J / U=0.1$, the dashed curve denoting the result for $U=\infty$. Circles express the experimental data of the enhancement ratio of $\gamma / \gamma_{0}$ in $\mathrm{Sr}_{1-x} \mathrm{La}_{x} \mathrm{TiO}_{3}$ [ref. 3], $\gamma_{0}$ being taken to be the $\gamma$ value at $N=0$.

Fig. 6 The electron number vs. interaction phase diagram of DHM (schematic), showing the paramagnetic metal (PM), paramagnetic insulator (PI), antiferromagnetic insulator (AFI) and ferromagnetic insulator (FI). The relative stability between AFI and FI at the quarter filling depends on the electronic and lattice structures and on the interaction of a given system. 


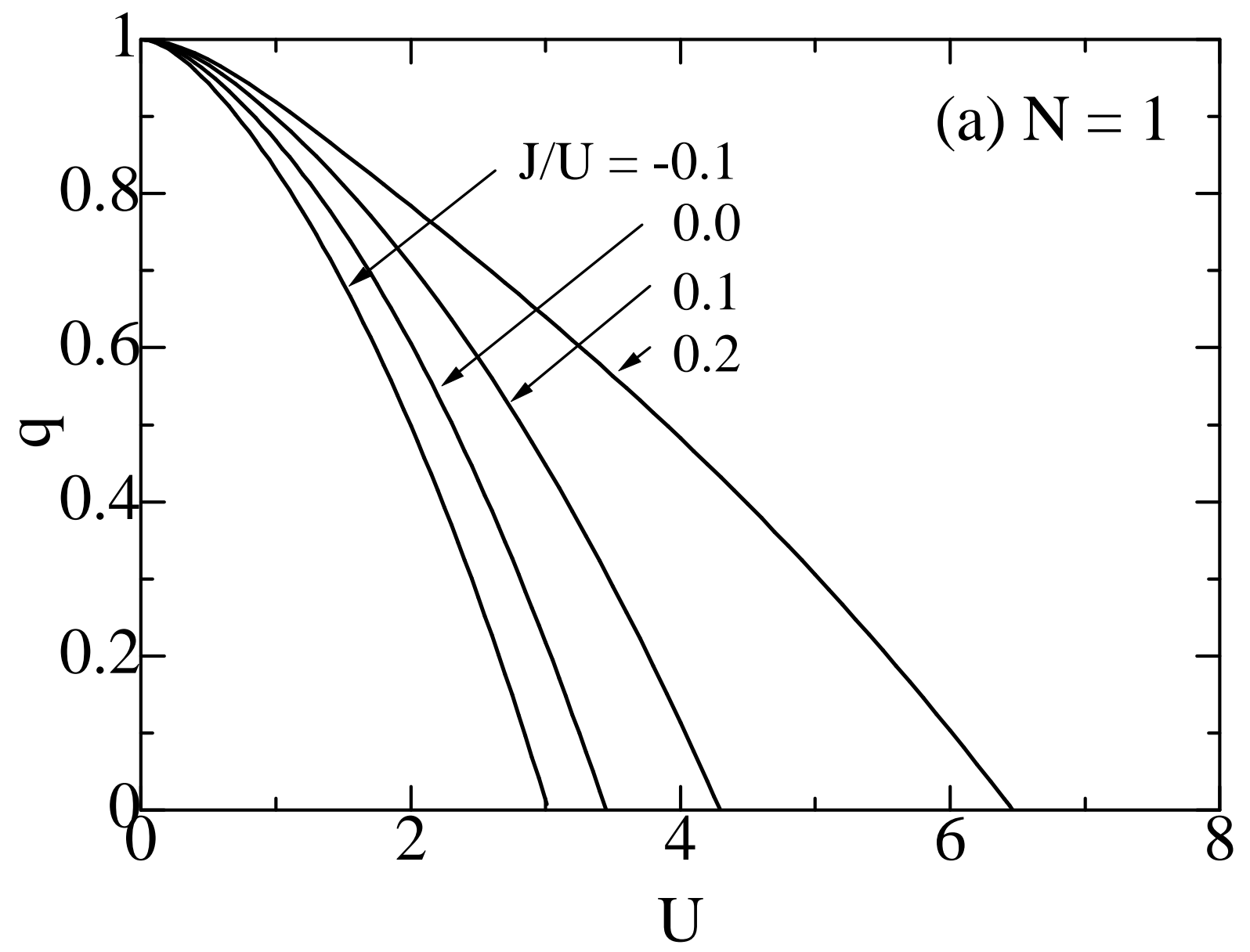




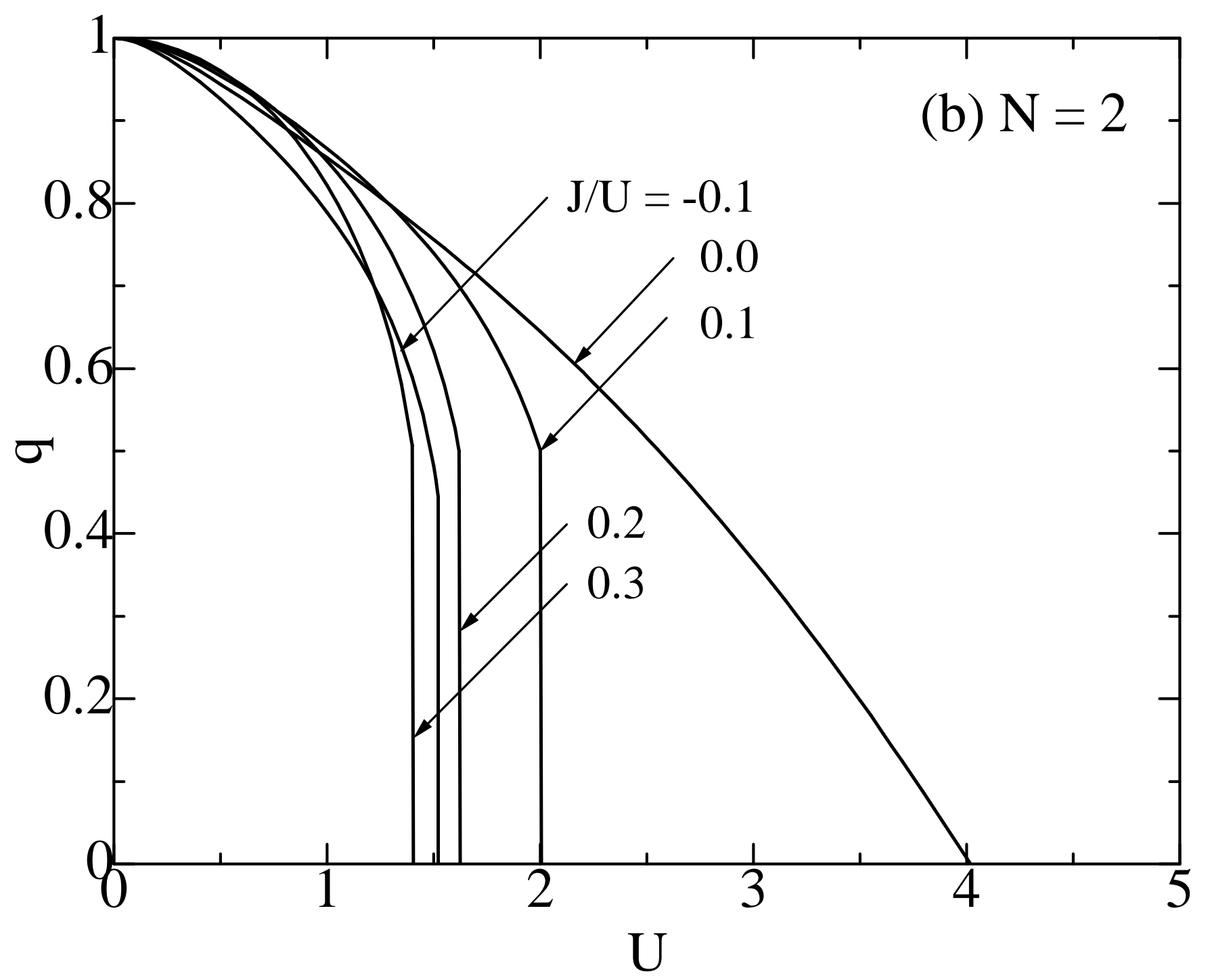




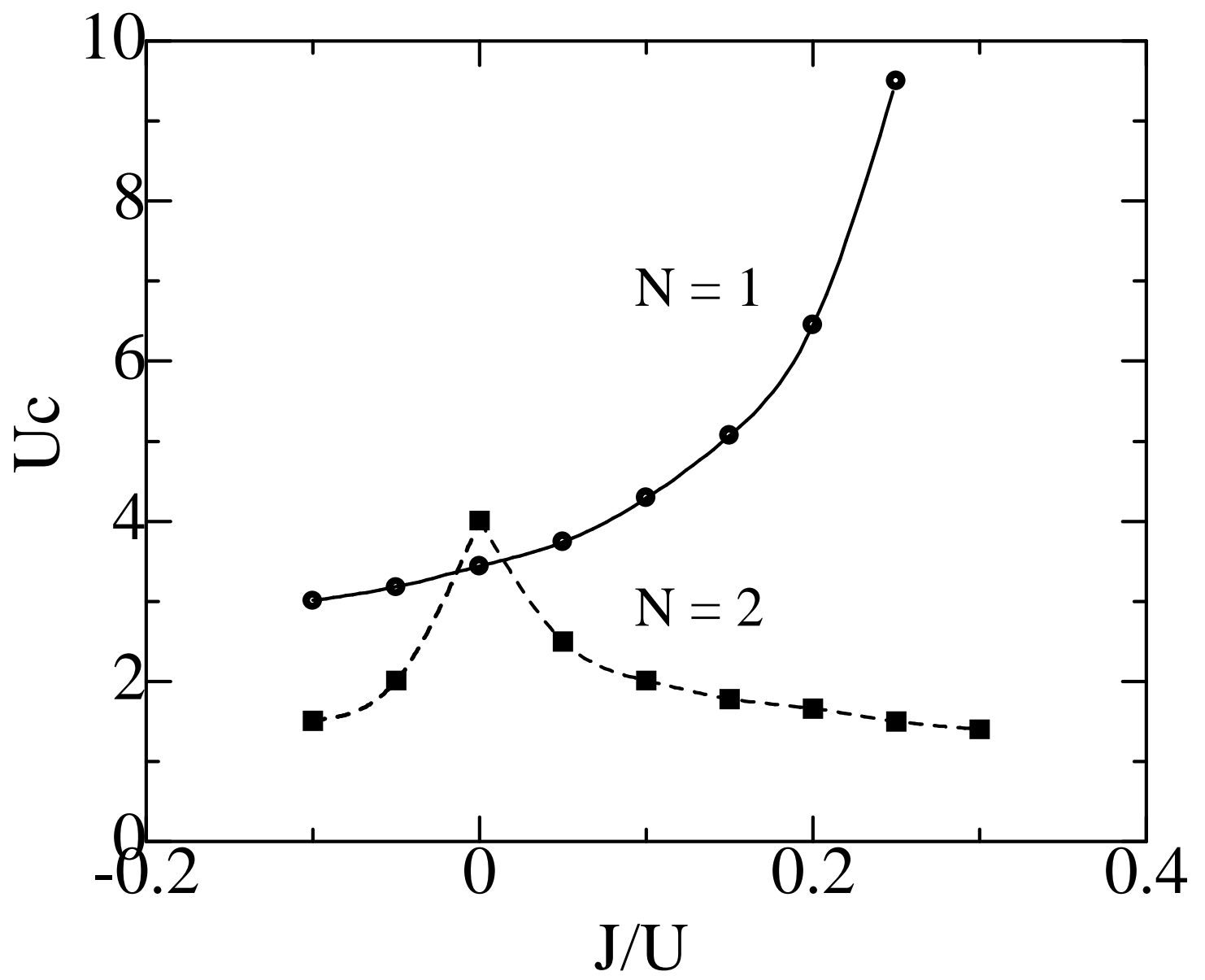




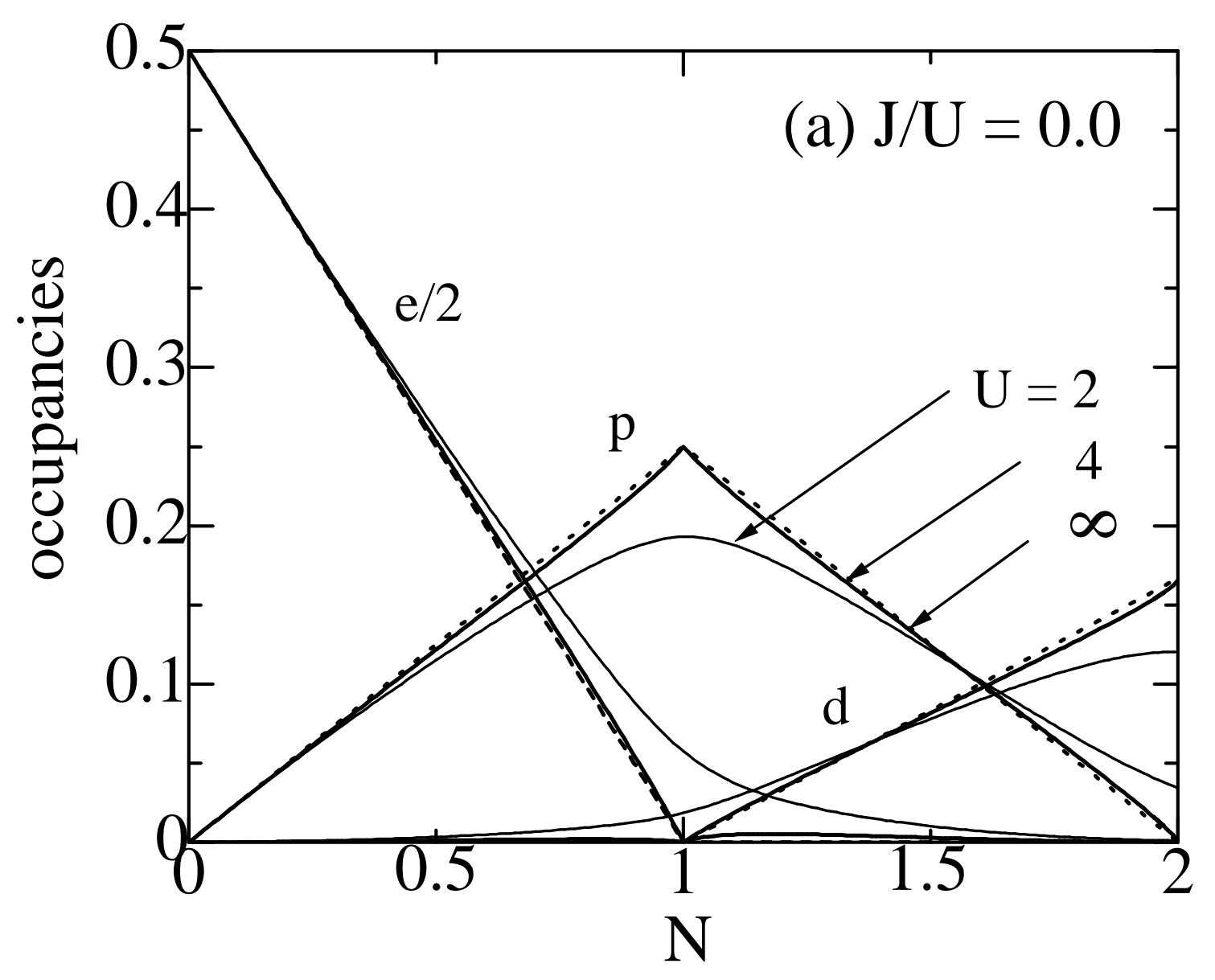




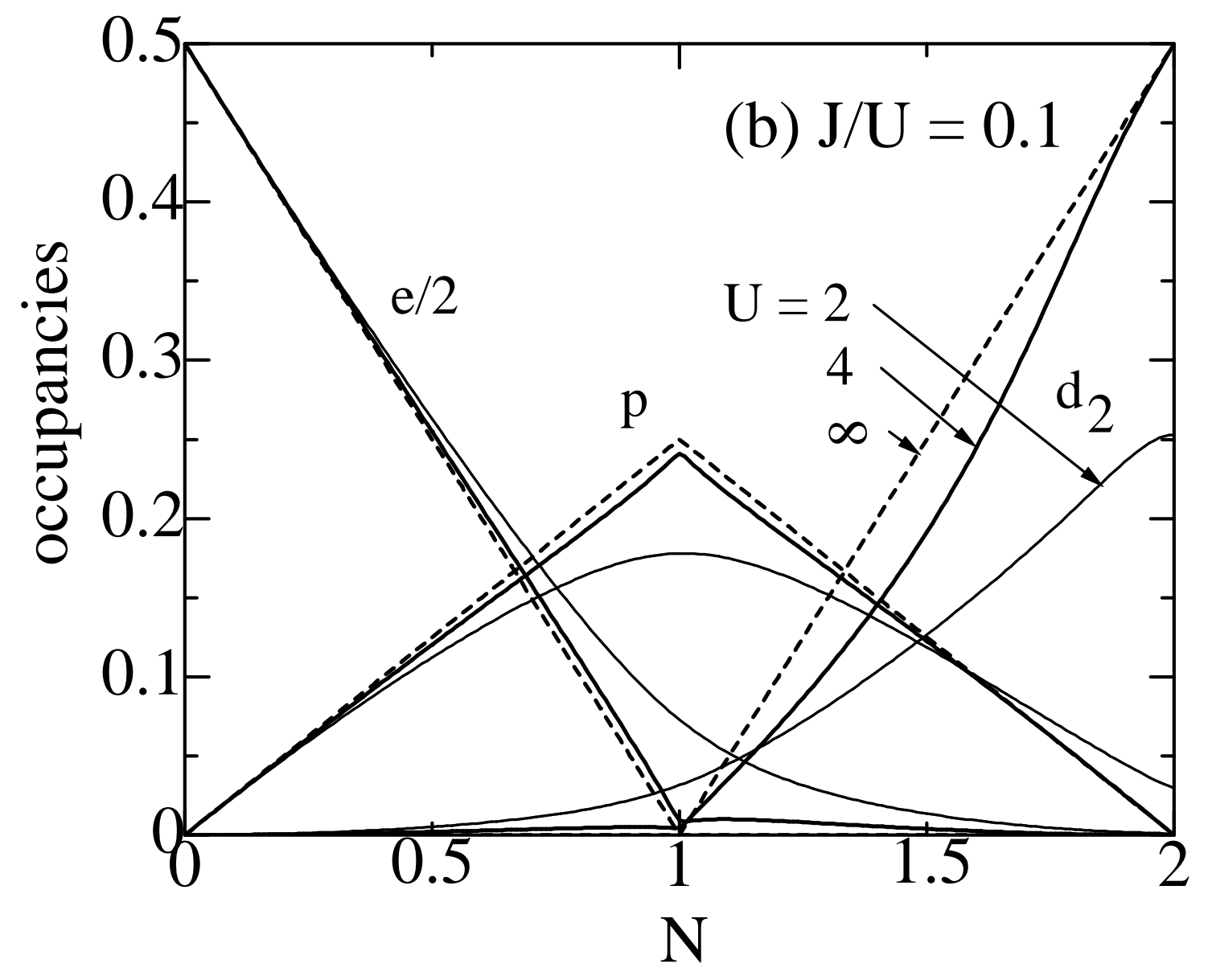




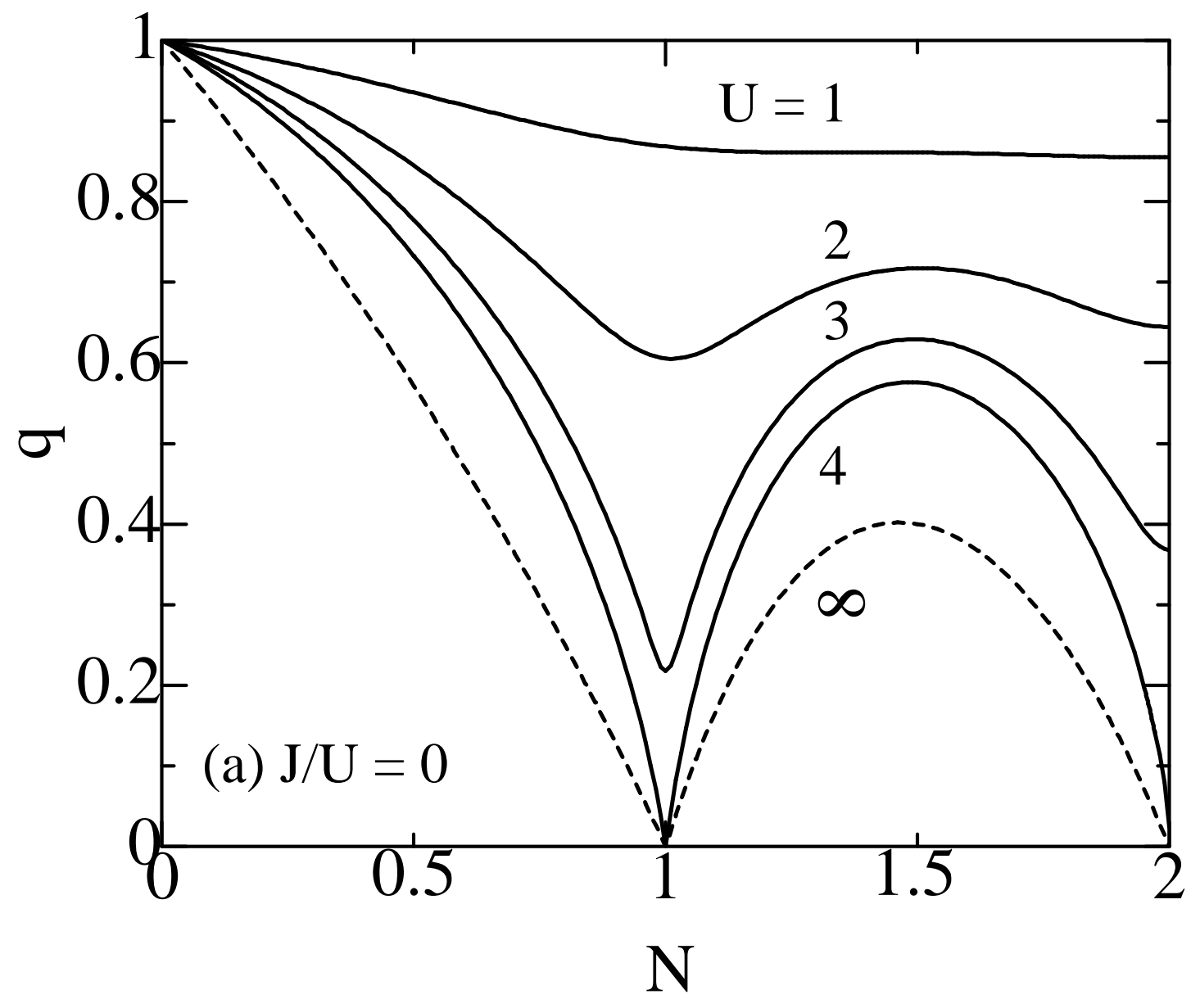




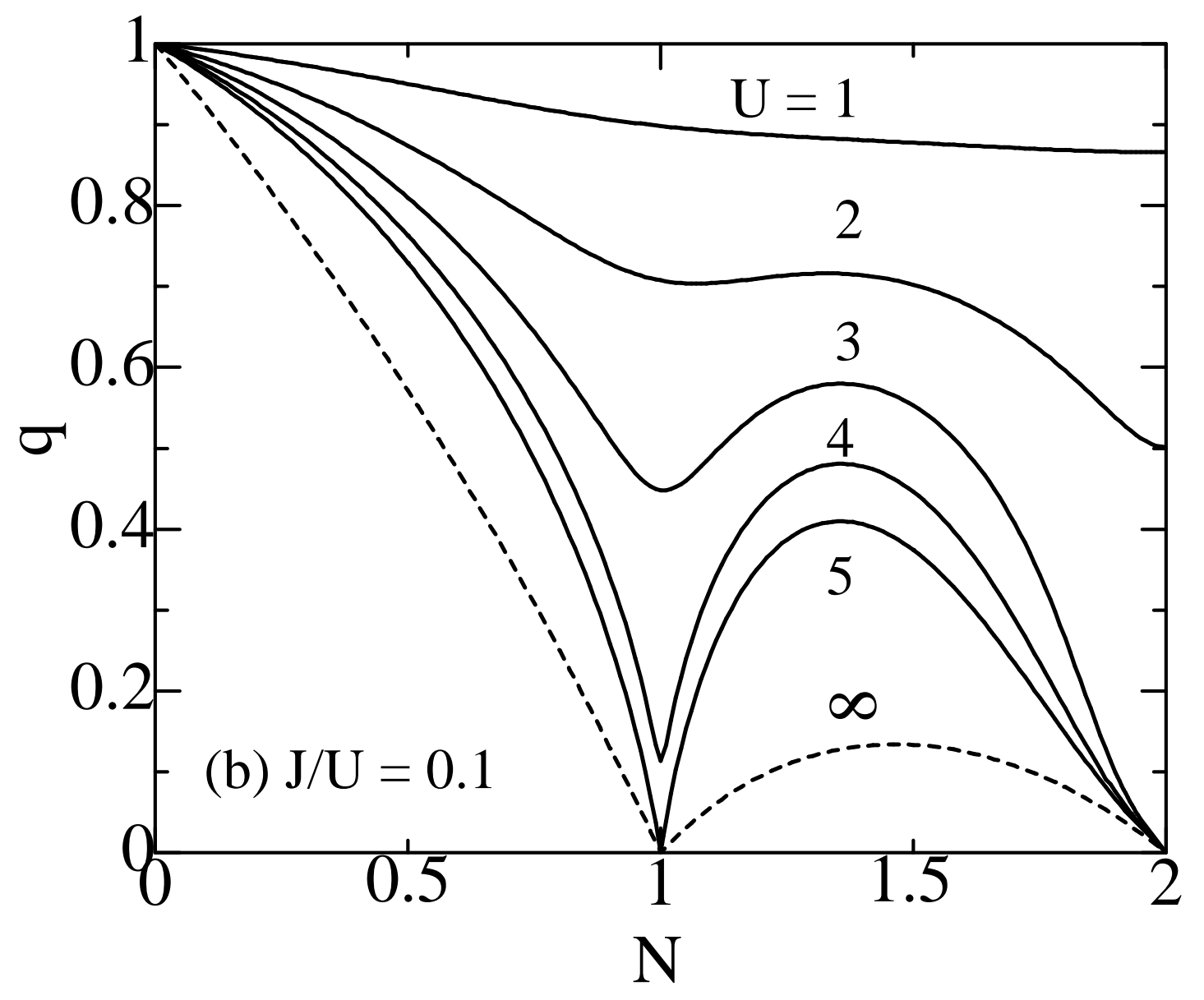




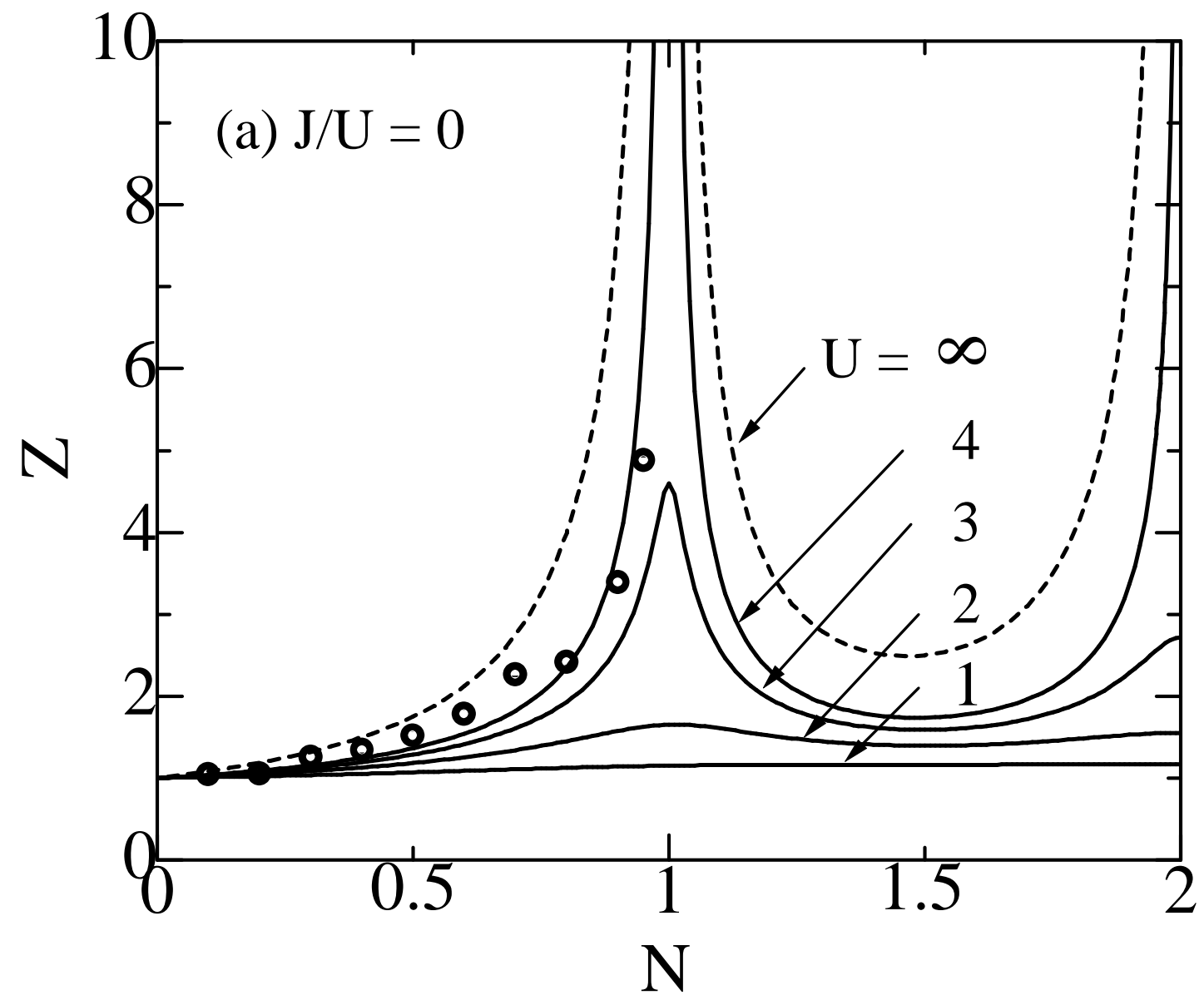




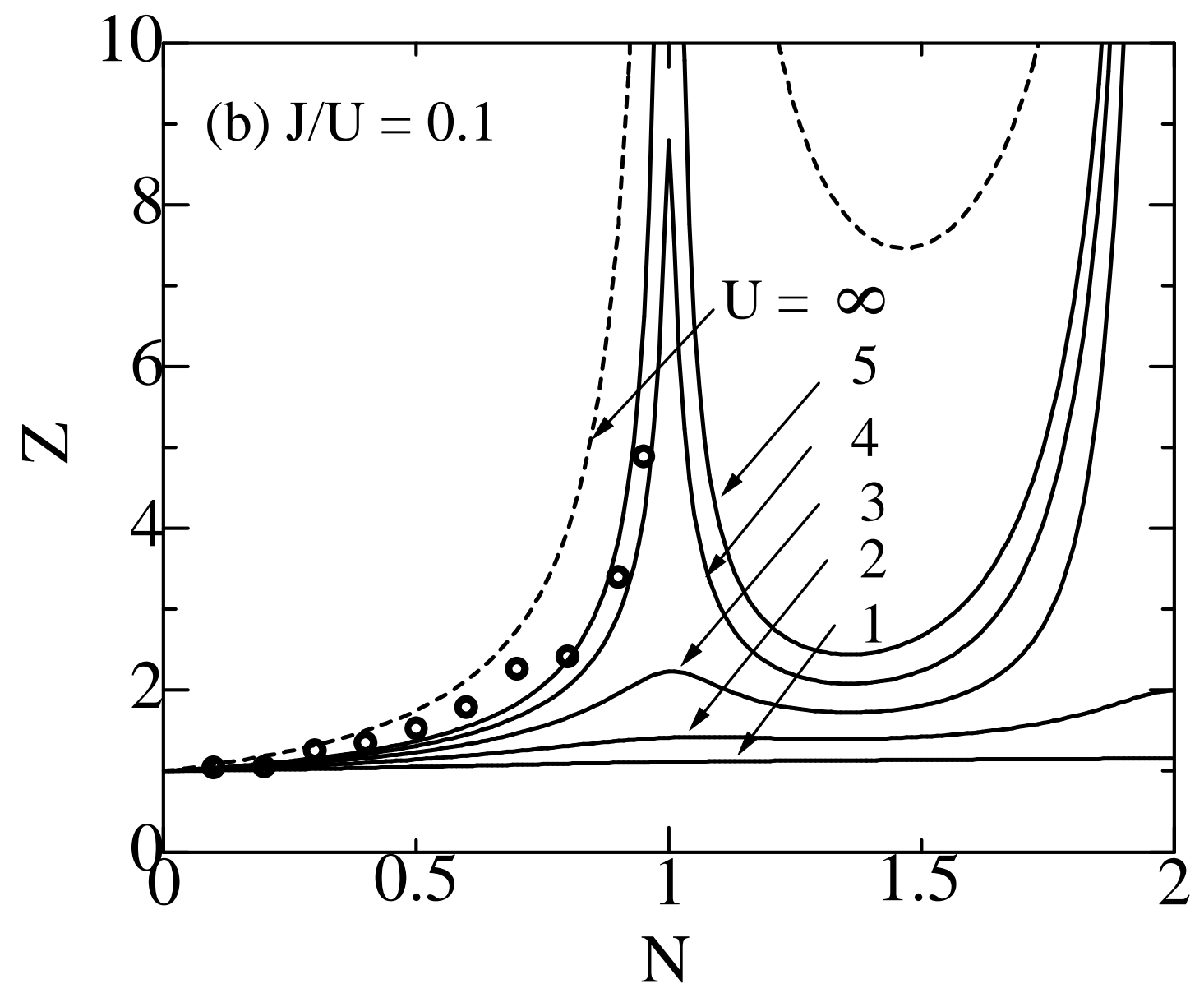




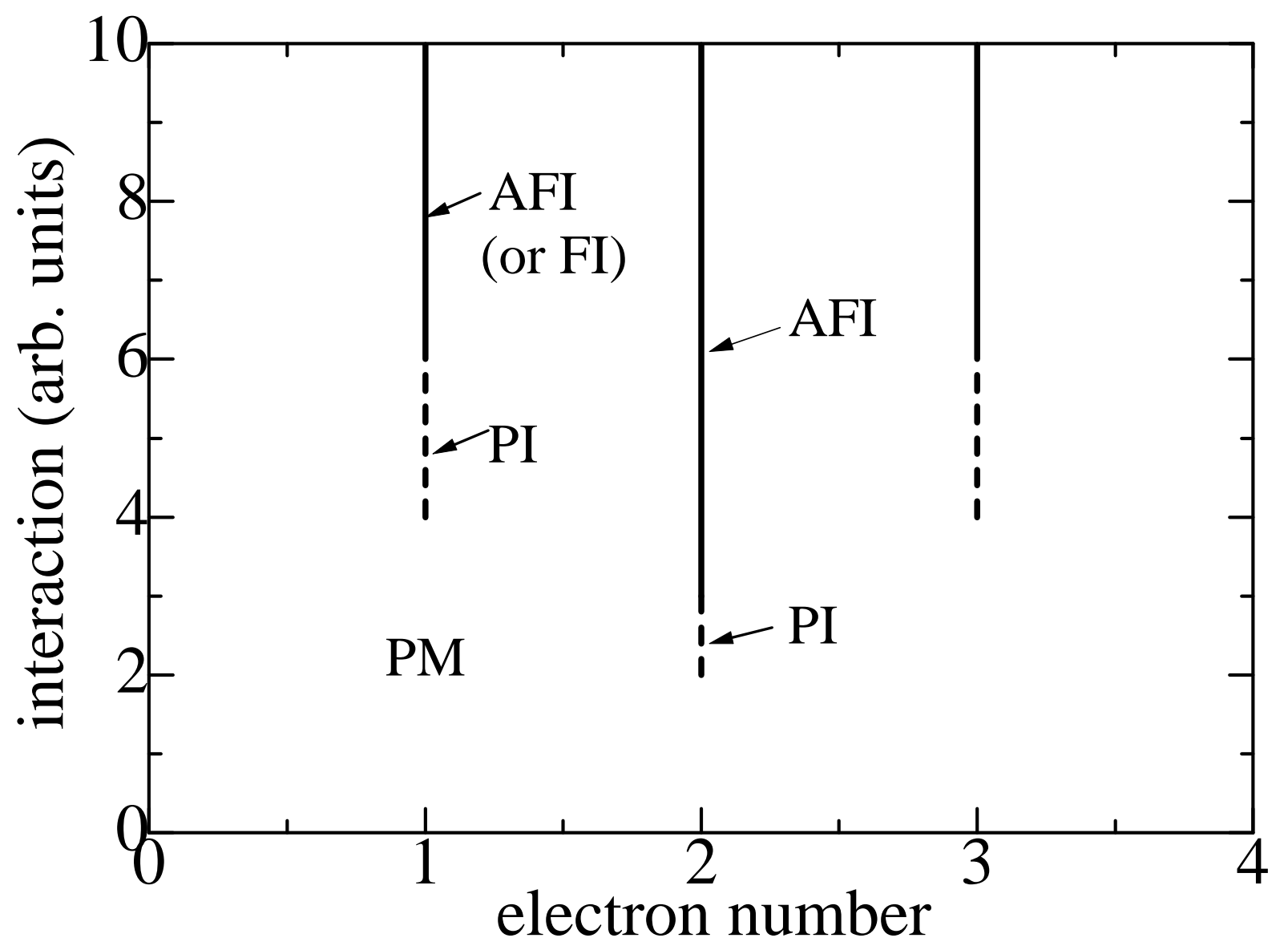

